\title{
Antitumor Effects of Conditional Replication Adenovirus in Combination with Cisplatin on Lung Cancer
}

\author{
Yanan Liu, Yinghui Huang \\ China-Japan Union Hospital ofJilin university, Jilin, China \\ Email: d10009@sina.com, yhuang@jlu.edu.cn
}

Received 2012

\begin{abstract}
Object: To explore the therapeutic effects and therapeutic mechanisms of Conditional Replication Adenovirus(CRAd) in combination with cisplatin on lung cancer cells. Methods: Using MTS / PMS assay, in vitro cell inhibition assay was performed to detect the cell viability of two lung cancer cell lines, NCI-H292 and NCI-H661. PCR was employed to detect the Coxsackie receptor(CAR) expression of cancer cells. The in vivo anti-tumor effect of CRAd and cisplatin was evaluated using a subcutaneous mouse model. Results: The CRAd with cisplatin is superior to the use of cisplatin or CRAd viruse alone on the suppression of lung cancer cell growth. The mechanism of inhibition is associated with the increased CAR expression. Conclusion: The application of CRAd in combination with cisplatin could play a better therapeutic effect on lung cancer cell growth inhibition.
\end{abstract}

Keywords: Lung Cancer; Adenovirus; Cisplatin

\section{Introduction}

Lung cancer is the second highest incidence of malignant tumors and the most common cause of cancer mortality [1]. Cisplatin (also known as DDP) is a chemotherpeutic drug often used to treat lung cancer, but serious side effects and drug resistance have severe impacts on clinical application [2,3]. CRAd (conditionally replication adenovirus) is able to specifically replicate and proliferate in tumor cells. When the tumor cells are lysised, then progeny virus will be released and infect the surrounding tumor cells. The adenovirus in combination with chemotherapy treatment of cancer has become an effective tool on cance treatment[4]. This experiment will explore the therapeutic effect and mechanism of CRAd combined with cisplatin in the treatment of lung cancer cell lines NCI-H292, NCI-H661 in vitro and in vivo.

\section{Methods}

\subsection{In vitro Cell Inhibition Assay}

NCI-H292, NCI-H661 cells were seeded in 24-well plates, at a density of $1 \times 10^{5}$ per culture well,after 24 hours, each well (a) treated three hours with different concentrations of cisplatin 0.25ug/ml, $1 \mathrm{ug} / \mathrm{ml}, 4 \mathrm{ug} / \mathrm{ml}, 16 \mathrm{ug} / \mathrm{ml}, 64 \mathrm{ug} / \mathrm{ml}$; (b) treated with different concentrations of CRAd virus 100MOI, 200MOI, 500MOI, 1000MOI, 2000MOI; (c) the treatment group each hole by adding 100MOI CRAd for four hours, and then different concentrations of cisplatin for three hours; (d) treated with each hole by adding different concentrations of cisplatin for three hours, then add 100MOI CRAd for four hours; different treated cells transferred to 96 -well plate, three wells, $5 \times 10^{3}$ cells per well, and observed for 5 days to add the MTS / PMS reagents,the absorbance at $490 \mathrm{~nm}$ was detected.

\subsection{Semiquantitative Reverse Transcription PCR}

After treated with cisplatin, NCI-H292 and NCI-H661 cells were collected, total RNA was extracted, each sample was converted to complementary cDNA, primer sequence is below:

GAPDH: S: 5'GATTGTTGCCATCAACGACC3 ' AS: 5 'GTGCAGGATGCATTGCTGAC 3' 371bp CAR: S: 5 'CCACCTCCAAAGAGCCGTAC 3' AS: 5 'ATCACAGGAATCGCACCC 3' 218bp

\subsection{Tumor Model}

BALB/C nude mice, female (6-8 weeks old) were acquired from the Chinese Academy of Sciences, NCI-H292, NCI-H661 cells $\left(4 \times 10^{6}\right)$ inoculated subcutaneous into the right flank of mice , Tumors were visible on the 15th day in NCI-H292 cell, NCI-H661 did not form tumors.

\subsection{In Vivo Tumor Inhibition Assay}

BALB/C nude mice, female (6-8 weeks old). NCI-H292 (8 x $\left.10^{6}\right)$, NCI-H661 $\left(8 \times 10^{6}\right)$ cells were inoculated subcutaneous into the right flank of mice ,tumor bearing mice( $n=6$ per group) were divided into three groups.Mice in each group were treated as follow: (a) cisplatin treatment group (b) CRAd treatment group (c) cisplatin combine CRAd treatment group (first give cislatin then give CRAd); Ad-luc virus transfected to each treatment group, a certain period of time to observe the effect by vivo imaging.

\section{Results}

1) Cisplatin could inhibit NCI-H292, NCI-H661 cell growth in a dose-dependent manner. No difference on the inhibition was found between the two cell lines

2) CRAd virus could significantly suppress the growth of NCI-H661 cells, but not on NCI-H292 cell line. 


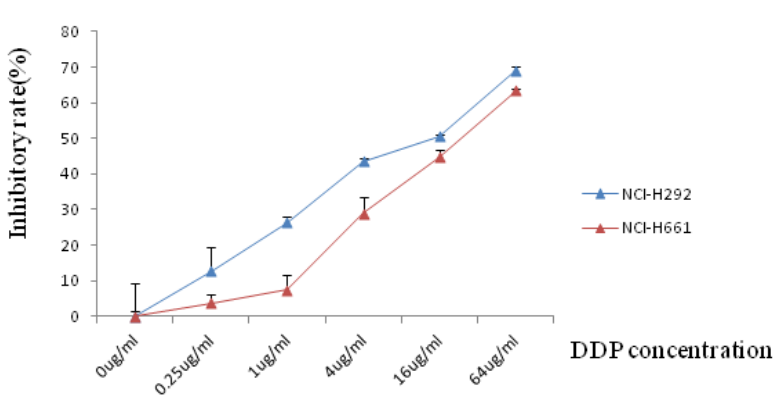

Figure 1. Inhibitory effects evaluated with MTS/PMS assay for the NCI-H292 and NCI-H661 cells treated with different concentration of DDP.

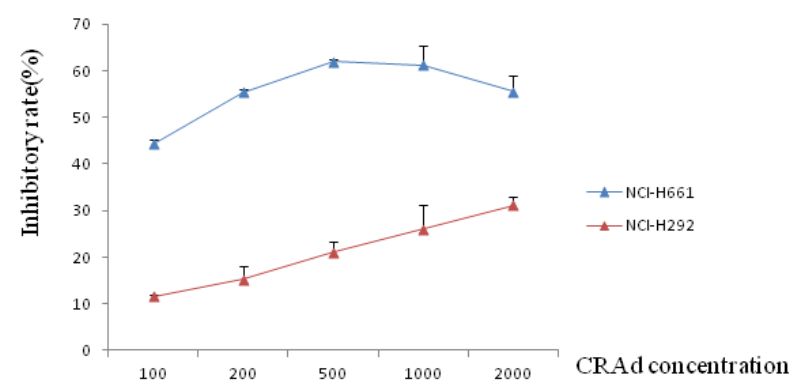

Figure 2. Inhibitory effects evaluated with MTS/PMS assay for the NCI-H292 and NCI-H661 cells treated with different concentration of CRAd.

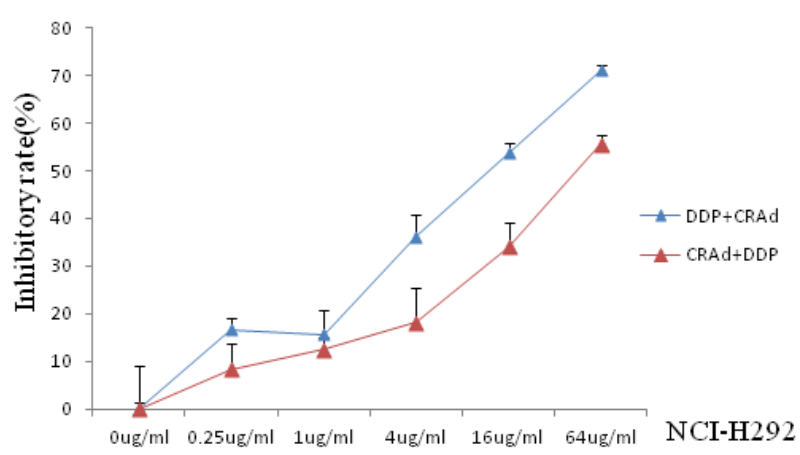

Figure 3. Inhibitory effects evaluated with MTS/PMS assay for the NCI-H292 cells treated with DDP and CRAd.

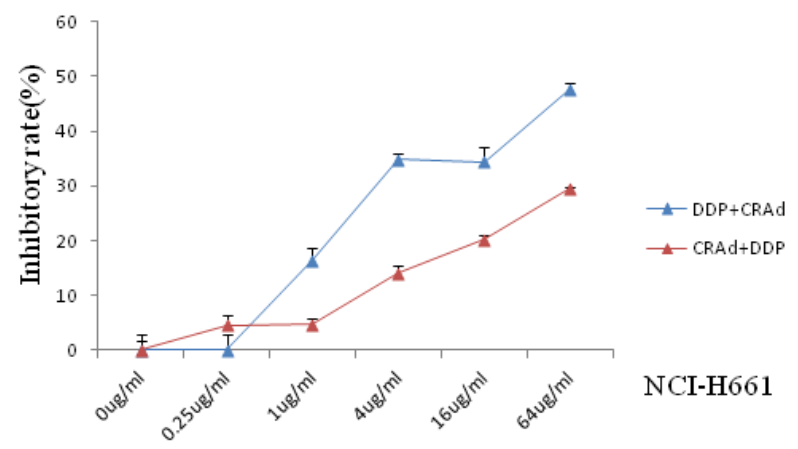

Figure 4. Inhibitory effects evaluated with MTS/PMS assay for the NCI-H661cells treated with DDP and CRAd.

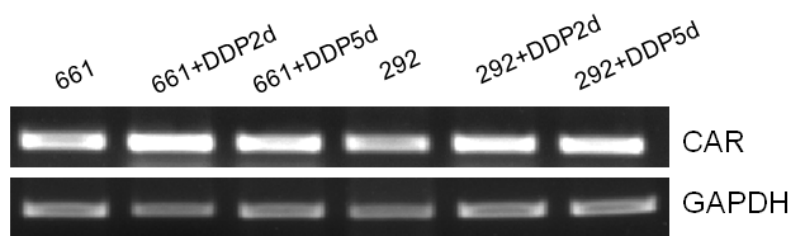

Figure 5. Gene expression of MDR was examined with PCR.

3) In combination with cisplatin and viral approach that MTS/PMS assay (d) is more obvious than the(c) inhibition of NCI-H292, NCI-H661 cells

4) PCR results showed that cisplatin promoted expression of CAR in the NCI-H292, NCI-H661 cells.

5) Tumor formation assay showed that NCI-H292 cell lines can form tumors, NCI-H661 cell lines is not easy to form a tumor.

6) The in vivo cell inhibition assay showed that enhanced inhibition of tumor growth was found in the group of cisplatin combined with CRAd virus.

\section{Discussion}

Our experimental results show that combined cisplatin and the virus is more effective than the separate application of cisplatin, or alone virus in the inhibition of tumor cell, the mechanism may be raised with cisplatin on the expression of adenovirus CAR receptor.The key aspects of adenoviral transduction is that adenovirus and the cell surface coxsackie adenovirus receptor (CAR) ${ }^{[5]}$ combining. Our experimental results show that the CAR of the cell surface receptor expression is enhanced by cisplatin, making the virus easier to invasive tumor cells, the combined treatment effects better than the simple .

Tumor in vivo experiments show that the transfer properties of the tumor cell line NCI-H292 more easily format tumor than without metastasis of cell line NCI-H661, in vivo inhibition of experimental results show that cisplatin combined CRAd virus group, inhibition of tumor growth and metastasis is the most effective. Its mechanism may still be raised coxsackie adenovirus receptor of tumor cells.

\section{REFERENCES}

[1] Siegel R, Naishadham D, Jemal A. Cancer statistics, 2012. CA Cancer J Clin. $2012: 62$ (1) :10-29.

[2] Arany I, Safirstein RL.Cisplatin nephrotoxicity.Semin NePhlol. 2003; 23 (5): 460 -464.

[3] Uyama N, Hatano E, Maetani Y, et al. Efficacy and toxicity of transcatheter arterial chemoembolization with cisplatin suspended inlipiodol for unresectable hepatocellularearcinoma.GanTo Kagaku Ryoho, 2008;35(5):775-780

[4] Chu RL, Post DE, Khuri FR,et al.Use of Replicating Oncolytic Adenoviruses in Combination Therapy for Caneer.Clinical Cancer Research .2004; 10(16): 5299-5312.

[5] Honda $\mathrm{T}$, Saitoh $\mathrm{H}$, Masuko $\mathrm{M}$, et al.The coxsackievirus adenovirus receptor protein as a cell adhesion molecule in the developing mouse brain $[\mathrm{J}]$.Brain Res Mol Brain Res, 2000,77:19-8. 